\title{
TÍCH HỢP BÀI TẬP MÔ PHỎNG VÀ ĐÁNH GIÁ THEO HÌNH THỨC HỔ SƠ TÀI LIỆ HỌC CÁ NHÂN VÀO VIẸC VIẾT THƯ TÍN THƯƠNG MẠI
}

\author{
Hứa Thị Tin ${ }^{a^{*}}$
}

${ }^{a}$ Khoa Ngoại ngũu, Trường Đại học Đà Lạt, Lâm Đồng, Việt Nam

Nhận ngày 03 tháng 12 năm 2015

Chỉnh sửa lần 1 ngày 15 tháng 03 năm 2016 | Chỉnh sửa lần 2 ngày 25 tháng 03 năm 2016

Chấp nhận đăng ngày 29 tháng 03 năm 2016

\section{Tóm tắt}

Bài viết này trình bày kết quả việc tích hợp bài tập mô phỏng (simulation tasks) và đánh giá theo hình thức hồ so tài liệu học cá nhân (portfolio assessment) vào việc viết thu tín thuoong mai trong các lớp tiếng Anh thuoong mai tại khoa Ngoai ngũu, trương Đại hoc Đà Lạt, đồng thời khảo sát thái độ của ngườ họ đối với phương pháp tích hợp này trong quá trình học viết thu tín thuong mại. Nghiên cứu này dự trên thuyết dạy học theo nhiệm vu (Task-based Language Teaching - viết tắt TBLT), sủ dụng bài tập mô phỏng, và thuyết đánh giá theo hình thức hồ so tài liệu học cá nhân. Kết quả nghiên cứu cho thấy việc tích hợp bài tập mô phỏng và đánh giá theo hình thức hồ so tài liệu học cá nhân dạng điện tử vào việc dạy viết thu tín thuoong mại đem lại một số lợi ich nhất định cho nguời học, tuy vẫn còn một vài hạn chế trong quá trình thực nghiệm và một vài vấn đề cần cân nhắc nếu muốn phuoong pháp này đem lại hiệu quả cao hơn.

Từ khóa: Dạy ngôn ngữ theo nhiệm vụ; Đánh giá theo hồ sơ tài liệu học cá nhân; Thư tín thương mại.

\section{GIỚI THIỆU}

Sinh viên ngành Anh văn thương mại hầu như không thể tiếp cận với thư tín thương mại của các công ty do yêu cầu bảo mật thông tin. Nhu cầu được sử dụng tiếng Anh thương mại trong môi trường làm việc thực tế của sinh viên khó được đáp ứng. Giáo trình về thư tín thương mại hiện đang sử dụng chứa nhiều tài liệu tham khảo, nhưng mang tính nặng về trình bày ngôn ngữ mẫu, các bài tập viết thư trong giáo trình tuy được thiết kế phù hợp với nội dung từng bài học, nhưng lại mang tính riêng lẻ, khó tạo sự hứng thú, chủ động của sinh viên trong việc học. Vì vậy cần có một phương pháp

\footnotetext{
*Tác giả liên hệ: Email: tinht@dlu.edu.vn
} 
tiếp cận tài liệu giáo trình theo hướng tạo sự chủ động tích cực cho sinh viên. Để giải quyết vấn đề này, hình thức bài tập mô phỏng (trong đó các nhóm sinh viên được phân vai khách hàng, nhà phân phối v.v., liên tục thực hiện giao tiếp bằng thư tín qua lại để hoàn tất một giao dịch thương mại) được đề xuất ứng dụng trong nghiên cứu này. Ngoài việc đánh giá bằng hình thức kiểm tra và thi cuối kỳ, đề tài tiến hành đánh giá quá trình học theo hình thức hồ sơ tài liệu học cá nhân dạng điện tử (e-portfolio) có tự nhận xét và nhận xét từ các sinh viên khác.

Mục đích của nghiên cứu này là thúc đẩy tính chủ động trong học tập của sinh viên, giúp sinh viên hình thành thói quen phân tích các tư liệu học tập thu thập được, thói quen làm việc nhóm, tư duy phản biện, đồng thời giúp sinh viên hình dung được phần nào sự tương tác giao tiếp liên tục trong quá trình hoàn thành một giao dịch thương mại trên thực tế. Qua đó sinh viên được trang bị kiến thức, kỹ năng cơ bản để tìm việc và làm việc trong lĩnh vực thương mại quốc tế.

Trong quá trình làm bài tập mô phỏng, sinh viên sẽ có động lực đọc, phân tích tài liệu trong giáo trình, tự tìm thêm tài liệu học tập bên ngoài, biết phân tích đánh giá các tư liệu học tập với hướng dẫn từ giáo viên, sau đó ứng dụng ngôn ngữ thu thập từ nhiều nguồn để hoàn thành một bài tập mô phỏng. Bài tập mô phỏng tạo điều kiện giúp sinh viên viết thư tín thương mại trong ngữ cảnh gần với thực tế và viết có mục đích, có yếu tố giao tiếp và tạo hứng thú học tập cho sinh viên. Hồ sơ tài liệu học cá nhân giúp sinh viên tự nhìn nhận, đánh giá được quá trình học của mình từ khi bắt đầu cho đến hết khóa học, thấy được những điểm mạnh, thiếu sót, và những tiến bộ trong quá trình học, từ đó có thể tự điều chỉnh việc học theo hướng tích cực. Hình thức hồ sơ tài liệu học cá nhân dạng điện tử (e-portfolio) giúp sinh viên dễ quản lý tài liệu học tập, dễ trao đổi eportfolio cho nhau để nhận xét, đánh giá, thúc đẩy sự tương tác giao tiếp giữa các thành viên trong nhóm. 


\section{CƠ SỞ LÝ LUẬN VÀ THỤ̉C TIẼ̃N}

\subsection{Thuyết dạy học theo nhiệm vụ}

Có nhiều phương pháp dạy học tiếng Anh, nhưng xu hướng dạy tiếng Anh được quan tâm rộng rãi hiện tại là giao cho sinh viên các nhiệm vụ được thiết kế phù hợp với việc phát triển một kỹ năng ngôn ngữ nào đó dựa theo thuyết TBLT (Đào, 2014; Nguyễn, 2014; Hu, 2013). Trong đó, dạng bài tập mô phỏng rất thích hợp cho việc phát triển kỹ năng viết thư tín thương mại vì dạng bài tập này giúp người học chủ động thu thập, tìm hiểu ngữ liệu liên quan để hoàn thành một nhiệm vụ cụ thể (Wang, 2003), và đồng thời tạo hứng thú cho người học qua những tình huống mô phỏng thực tế, từ đó phát triển các kỹ năng cần thiết trong việc xử lý các loại văn bản trong công việc (Sampath \& Zalipour, 2009).

Bài tập mô phỏng (simulation task) là một trong những dạng bài tập tác vụ (task), nên chia sẻ những đặc điểm chung của một task. Có rất nhiều định nghĩa khác nhau về task. Theo Lee (2000), task là một dạng bài tập hay hoạt động có mục đích, mục đích này chỉ có thể đạt được thông qua sự tương tác giữa các học viên, có cơ chế để liên kết các hoạt động tương tác một cách có trình tự, có trọng tâm là trao đổi thông tin. Ngoài ra task còn là động lực để học ngôn ngữ: người học cần hiểu, thao tác, sản sinh ngôn ngữ đích trong quá trình thực hiện task. Theo Ellis (2003), một task đúng nghĩa cần phải có: mục đích cụ thể, ngữ liệu đầu vào, điều kiện thực hiện, phương thức thực hiện, sản phẩm, quá trình lĩnh hội ngôn ngữ và quá trình nhận thức.

Tích hợp các quan điểm phổ biến về task như trên, nghiên cứu này đưa ra một định nghĩa chung về task, và định nghĩa này được dùng làm tham chiếu để chọn lọc, chỉnh sửa các bài tập mô phỏng sao cho phù hợp với người học, điều kiện lớp học và phù hợp với nhu cầu phát triển ngôn ngữ. Theo đó, các bài tập mô phỏng trong nghiên cứu này cần đảm bảo những yêu cầu sau: (1) có mục đích cụ thể, mục đích này chỉ có thể đạt được thông qua sự tương tác giữa các học viên, (2) có cơ chế để liên kết các hoạt động tương tác một cách có trình tự, thể hiện qua việc người học được cung cấp đầy đủ hướng dẫn cần thiết, (3) có trọng tâm là trao đổi thông tin, (4) có ngữ liệu đầu vào, (5) đảm bảo điều kiện thực hiện, (6) có phương thức thực hiện phù hợp, (7) có sản phẩm (kết quả dự kiến), (8) là động lực để học ngôn ngữ: người học cần hiểu, thao tác, sản sinh ngôn ngữ đích trong quá trình thực hiện task, (9) gắn kết việc học ngôn ngữ trong lớp với việc sử dụng ngôn ngữ bên ngoài lớp thông qua việc lựa chọn task có nội dung 
phù hợp, và giới thiệu các tài liệu thực tế vào quá trình học tập, (10) thúc đẩy quá trình nhận thức, tạo cơ hội trải nghiệm quá trình học.

\subsection{Thuyết đánh giá quá trình học theo hình thức hồ sơ tài liệu học cá nhân}

Việc sử dụng hồ sơ tài liệu học cá nhân (portfolio) cũng được áp dụng nhiều trong các lớp ngôn ngữ gần đây như là một hình thức đánh giá hiệu quả liên tục quá trình học tập, lĩnh hội ngôn ngữ, đặc biệt là trong việc phát triển kỹ năng viết (Jeevaratnam, 2013). Hình thức này lấy người học làm trung tâm, và nhấn mạnh vào quá trình học, chứ không chỉ là vào kết quả cuối cùng (Hamp-Lyons \& Condon, 1998). Archibald \& Newmann (1992) định nghĩa portfolio là một tập hợp đa dạng các tư liệu cung cấp thông tin về quá trình học, những kinh nghiệm và các kết quả học tập của người học theo thời gian. Hồ sơ tài liệu học cá nhân là thước đo khả năng sử dụng ngôn ngữ, đồng thời đảm bảo tính thực dùng ngôn ngữ trong ngữ cảnh cụ thể, phù hợp với với phương pháp dạy ngôn ngữ qua giao tiếp (communicative language teaching approach) (Milanovic \& Saville, 1996).

Dựa theo lý thuyết về portfolio assessment của Hamp-L.yons và Condon (1998), Cambridge (2001) đã đưa ra bốn đặc trưng của e-portfolio như sau: (1) thể hiện đa dạng các bài tập, (2) có bối cảnh rõ ràng: nghĩa là phải có phân công công việc cụ thể, hướng dẫn làm bài, giới thiệu các tiêu chí đánh giá e-portfolio, kỹ thuật đánh giá, tương tác ngoài lớp học (3) cho người học cơ hội tự lựa chọn, tự đánh giá trong quá trình học, (4) phản ánh quá trình học liên tục theo thời gian, từ đó có thể cập nhật những điều chỉnh cần thiết.

Một nghiên cứu gần đây do trường đại học York St John University (Anh) thực hiện trong năm học 2011-2012 nhằm đánh giá hiệu quả của việc sử dụng e-portfolio vào việc dạy ngoại ngữ (Ferrari \& Zhurauskaya, 2012). Nghiên cứu này cho thấy ý kiến sinh viên phản hồi việc sử dụng e-portfolio vào dạy ngoại ngữ là tích cực, vì giúp sinh viên yêu thích việc học và tích cực học tập hơn. Nghiên cứu của Ferrari và Zhurauskaya cũng lưu ý việc sử dụng e-portfolio cần phải dễ dàng đối với người học và người dạy, và nhấn mạnh e-portfolio không phải là hình thức portfolio giấy chuyển vào máy vi 
tính, mà là một công cụ đánh giá hoàn toàn khác, cung cấp cho người học một môi trường học tập tự chủ hơn.

Mục đích chính của việc dạy viết thư tín thương mại là trang bị cho người học kỹ năng viết hiệu quả để thực hiện nhiệm vụ kinh doanh. Ngoài yếu tố viết đúng nội dung, đúng hình thức, văn phong phù hợp với ngữ cảnh, người học cần viết nhanh, trong thời gian ngắn thì mới đáp ứng được yêu cầu công việc. Mô hình $5 C$ 's hướng dẫn viết thư tín thương mại do Jones \& Alexander (2000) đề xuất liệt kê khá đầy đủ các đặc trưng cơ bản của thư tín thương mại, đó là complete (đủ thông tin cần thiết), concise (súc tích, ngắn gọn), clear (rõ ràng), courteous (lịch sự, chân thành), và correct (đúng ngữ pháp, chính tả, dấu câu). Mô hình trên có thể được xem là các quy tắc hướng dẫn viết thư thương mại sao cho hiệu quả, và nghiên cứu này ứng dụng mô hình $5 C$ 's này như là một tham chiếu để sinh viên định hướng trong quá trình viết, và là các tiêu chí để đánh giá các bài viết trong e-portfolio.

\section{PHƯƠNG PHÁP NGHIÊN CÚU}

\subsection{Khách thể nghiên cứu}

Khách thể nghiên cứu trong bài viết này là sinh viên năm tư chuyên ngành Tiếng Anh Thương Mại, trường Đại học Đà Lạt. Sinh viên được chia thành 2 nhóm: nhóm thực nghiệm (experimental group) gồm 30 sinh viên và nhóm đối chứng (control group) gồm 35 sinh viên. Thời gian nghiên cứu kéo dài một học kỳ. Chương trình dành chung cho hai nhóm đối tượng là Tiếng Anh Thương Mại 4: Thương mại quốc tế (Business English 4: International Trade).

\section{2. Đối tượng nghiên cứu}

Nghiên cứu này được tiến hành nhằm đánh giá tính hiệu quả của việc tích hợp bài tập mô phỏng và đánh giá theo hình thức hồ sơ tài liệu học cá nhân dạng điện tử vào việc dạy viết thư tín thương mại, và kết quả khảo sát thái độ của người học đối với việc áp dụng này, dựa trên cơ sở lý thuyết về TBLT và portfolio assessment. 


\subsection{Công cụ nghiên cứu}

Ngoài các bài tập trong giáo trình, nhóm thực nghiệm còn được yêu cầu làm thêm các bài tập mô phỏng, mỗi bài tập mô phỏng gồm nhiều bước nhỏ (stage), kéo dài bốn tuần, các bài tập mô phỏng này yêu cầu sinh viên phải viết nhiều loại thư tín thương mại để hoàn thành một giao dịch và được trình bày trong e-portfolio. Các bài tập mô phỏng trong nghiên cứu này được lấy từ sách Company to Company: A task-based approach to business emails, letters and faxes của Littlejohn (2005) và được thiết kế lại cho phù hợp với mục đích nghiên cứu. Điểm e-portfolio chiếm $20 \%$ tổng số điểm môn học để tạo động lực cho sinh viên hoàn thành bài tập mô phỏng và e-portfolio. Đối với nhóm đối chứng, điểm $20 \%$ tương ứng này là cho việc hoàn thành các bài tập riêng lẻ nằm trong giáo trình.

Trong nghiên cứu này, Google Docs được sử dụng để tạo e-portfolio cho sinh viên. Goolge Docs được chọn bởi những ưu điểm sau: (1) giáo viên có thể tạo các khuôn mẫu (template) để sinh viên nhập dữ liệu dễ dàng, (2) Google docs cho phép sinh viên dàn trang khi viết thư, email, fax trong thương mại, vốn có yêu cầu riêng về hình thức thư tín thương mại (blocked style), (3) sinh viên chỉ cần có hộp thư gmail thì có thể đăng nhập dễ dàng, (4) việc đăng bài, tự chỉnh sửa, chỉnh sửa và đánh giá chéo giữa các sinh viên dễ dàng tiện lợi, (5) giáo viên có thể quản lý, theo dõi quá trình thực hiện eportfolio của sinh viên liên tục, và có những điều chỉnh kịp thời, tránh tình trạng sinh viên làm không đúng theo yêu cầu, (6) việc cập nhật thêm thông tin, tư liệu dễ thực hiện, (7) các chức năng Revision history, Share, Comments, Mode của Google Docs giúp giáo viên thiết lập và quản lý thời gian nộp bài, cũng như dễ theo dõi quá trình chỉnh sửa và đánh giá chéo giữa các sinh viên.

\subsection{Câu hỏi nghiên cứu}

Để đánh giá tính hiệu quả của phương pháp đề xuất, các câu hỏi nghiên cứu sau đây được đưa ra:

- Có sự khác biệt nào về kết quả học tập của sinh viên sau khi tích hợp bài tập mô phỏng và đánh giá theo hình thức hồ sơ tài liệu học cá nhân dạng điện tử vào 
việc dạy viết thư tín thương mại, so với phương pháp dạy viết thư thương mại truyền thống hiện đang được dùng?

- Các bài tập mô phỏng có thú vị, tạo động lực học đối với sinh viên không?

- Hình thức đánh giá e-portfolio có giúp sinh viên liên tục tự đánh giá quá trình học của bản thân mình, qua đó cải thiện và củng cố kiến thức không?

Để trả lời các câu hỏi được nêu ra ở trên, nghiên cứu này đưa ra các giả thuyết và dùng kết quả kiểm tra tiền thực nghiệm (Pre-test) và kiểm tra sau thực nghiệm (Post test), cùng kết quả khảo sát ý kiến sinh viên để kiểm chứng những giả thuyết sau:

- Giả thuyết 1 : Phương pháp đề xuất giúp sinh viên tích lũy vốn từ chuyên ngành thương mại, và phát triển kỹ năng viết các loại thư tín thương mại, qua đó học tốt hơn và tăng điểm sau quá trình học.

- Giả thuyết 2: Việc áp dụng phương pháp đề xuất giúp sinh viên tăng điểm nhiều hơn so với không áp dụng phương pháp đề xuất.

- Giả thuyết 3: Sinh viên yêu thích các bài tập mô phỏng và đồng ý rằng việc tích hợp các bài tập mô phỏng và e-porfolio vào việc học viết thư tín thương mại là có hiệu quả.

- Giả thuyết 4: Phương pháp đề xuất giúp sinh viên tăng khả năng tự học, tự nghiên cứu, làm việc nhóm, tạo thói quen liên tục đánh giá chiêm nghiệm quá trình học của bản thân mình.

\subsection{Phương thức thu thập dữ liệu}

Để kiểm chứng Giả thuyết 1 và Giả thuyết 2 nêu trên, nghiên cứu này tiến hành cho sinh viên nhóm thực nghiệm và nhóm đối chứng làm bài kiểm tra trước và sau thực nghiệm (Pre-test và Post-test). Để kết quả Post-test đáng tin cậy hơn, nghiên cứu này đã cho sinh viên làm hai Post-test: Post-test 1 và Post-test 2 . Post-test 1 được thực hiện sau 
khi sinh viên học được khoảng một nửa chương trình, và Post-test 2 được thực hiện sau khi sinh viên học hết học phần Tiếng Anh thương mại 4.

Để kiểm chứng Giả thuyết 3 và Giả thuyết 4 nêu trên, một bảng câu hỏi khảo sát bao gồm 14 câu hỏi đóng (closed question), và 2 câu hỏi mở (open question) được gửi đến sinh viên sau khi kết thúc khóa học, nhằm để tìm hiểu thái độ của người học đối với việc sử dụng bài tập mô phỏng và e-portfolio, những điều hài lòng và những khó khăn mà sinh viên gặp phải. Ngoài ra một phỏng vấn được thực hiện với năm em nằm trong năm nhóm khác nhau với mong muốn có thể thu thập được ý kiến phổ quát của toàn bộ sinh viên tham gia thực nghiệm. Bảng câu hỏi phỏng vấn nhằm đi sâu tìm hiểu những khó khăn mà sinh viên có thể gặp phải trong quá trình thực hiện bài tập mô phỏng và eportfolio.

\subsection{Cách thức phân tích dữ liệu}

Nghiên cứu tiến hành so sánh điểm trung bình kiểm tra giữa Pre-test, Post-test 1 và Post-test 2 , giữa nhóm thực nghiệm và nhóm đối chứng. Kết quả nghiên cứu dựa trên kết quả điểm của 27 sinh viên trong nhóm thực nghiệm và 33 sinh viên trong nhóm đối chứng do có 3 sinh viên trong nhóm thực nghiệm và 2 sinh viên trong nhóm đối chứng không tham dự pre-test hoặc post-test. Để kiểm tra kết quả thực nghiệm của phương pháp đề xuất có ý nghĩa thống kê, phương pháp t-test được sử dụng.

Kết quả khảo sát ý kiến sinh viên bằng bảng câu hỏi khảo sát và phỏng vấn sau khi kết thúc học phần được phân tích định lượng và định tính, nhằm để tìm hiểu thái độ của người học đối với việc sử dụng bài tập mô phỏng và e-portfolio, mức độ hài lòng và những khó khăn mà sinh viên gặp phải trong quá trình thực nghiệm. Dựa trên kết quả phân tích định lượng và định tính, một số đề xuất được đưa ra nhằm giúp cho việc tích hợp bài tập mô phỏng và e-portfolio vào việc dạy viết thư tín thương mại đạt hiệu quả hơn. 


\subsection{Cách thức tích hợp bài tập mô phỏng và đánh giá theo hình thức hồ sơ tài liệu học cá nhân dạng điện tử vào việc dạy viết thư tín thương mại ở nhóm thực nghiệm}

Để áp dụng TBLT, các nhà nghiên cứu đã đưa ra các mô hình hướng dẫn của phương pháp này. Trong đó mô hình hướng dẫn phương pháp dạy học TBLT do Willis (1996) đề xuất được nhiều học giả tán đồng nhất và là mô hình rõ ràng nhất (Nguyễn, 2014). Nghiên cứu này áp dụng mô hình do Willis (1996) đề xuất bên trên, vì theo như Nguyễn (2014) đánh giá, mô hình này nêu rõ cụ thể những nhiệm vụ mà giáo viên và người học phải thực hiện ở mỗi giai đoạn, dễ thực hiện, không chỉ tăng cường kỹ năng giải quyết nhiệm vụ và khả giao tiếp trôi chảy (fluency) mà còn quan tâm tới sự chính xác (accuracy). Việc áp dụng mô hình này có một vài thay đổi, để phù hợp với tình hình thực tế của lớp học và với mục đích nghiên cứu. Sau đây là các giai đoạn thực hiện simulation task trong chương trình thực nghiệm:

\section{Giai đoạn trước khi thụ̣c hiện Task (Pre-task):}

Trước khi sinh viên (SV) thực làm simulation task, giáo viên (GV) dành 1 buổi giới thiệu, hướng dẫn $\mathrm{SV}$ cách thực hiện. Buổi giới thiệu nên được tiến hành trong phòng máy tính, bao gồm 4 bước như sau:

Bước 1: GV phân $30 \mathrm{SV}$ trong nhóm thực nghiệm thành 5 nhóm, mỗi nhóm gồm 6 thành viên. Mỗi nhóm lại phân ra làm ba cặp (Pair) gồm Pair A, Pair B, và Pair $\mathrm{C}$, lần lượt đóng vai nhà cung cấp và khách hàng.

Bước 2: Giới thiệu SV sơ đồ chia e-portfolio để đánh giá theo nhóm, cặp.

Bước 3: Giới thiệu SV các tiêu chí đánh giá e-portfolio, và các tiêu chí để SV biết cách phác thảo, chỉnh sửa và đánh giá một bài viết trong e-portfolio. Thang điểm này được xây dựng dựa trên thuyết $\mathrm{TBLT}$ và mô hình $5 C^{\prime} s$ hướng dẫn viết thư tín thương mại do Jones \& Alexander (2000) đề xuất. 
Bước 4: Giới thiệu chung về simulation task cần thực hiện, giúp SV hiểu bối cảnh và yêu cầu của task. Phân vai cho các cặp trong mỗi nhóm. Để thuận tiện, các bản mẫu (template) cho e-portfolio của SV đã được tạo sẵn.

Nội dung của simulation task được chọn lọc, chỉnh sửa sao cho phù hợp với nội dung học trong chương trình, ứng với thứ tự các chương trong giáo trình, đảm bảo $\mathrm{SV}$ liên tục viết các loại thư tín thông dụng trong các giao dịch quốc tế. GV thông báo $\mathrm{SV}$ nên đọc tham khảo những chương nào trong giáo trình để hỗ trợ $\mathrm{SV}$ phần ngữ liệu (input), giải thích các thuật ngữ chuyên ngành, cung cấp thêm tư liệu thực dùng (authentic materials). GV khuyến khích sinh viên tìm thêm tư liệu bên ngoài giáo trình có nội dung liên quan đến vấn đề đang học, và đưa toàn bộ tài liệu tham khảo này vào eportfolio dưới dạng các đường link trong phần Additional Materials ở cuối mỗi eportfolio.

Giai đoạn thực hiện Task (Task Cycle) bao gồm 3 bước như sau:

- Doing Task (Thực hiện task): SV thực hiện tuần tự các stage trong simulation task theo nhóm. Các e-portfolio định dạng sẵn giúp SV nắm rõ vai mình đóng, yêu cầu công việc cho từng vai, và ai là người mình cần trao đổi thông tin. $G \mathrm{~V}$ chỉ giám sát, và khuyến khích $\mathrm{SV}$ trao đổi, gởi email nhắc nhở khi thấy nhóm/cặp nào chậm trễ làm bài, không chỉnh sửa bài viết vì trong giai đoạn này SV cần tự do thử nghiệm ngôn ngữ.

- Planning (Lập kế hoạch): SV chuẩn bị công bố các thư tín đã viết trong quá trình thực hiện task. Trong giai đoạn này, các nhóm trao đổi e-portfolio, và cho nhận xét, gợi ý chỉnh sửa đối với các bài viết và với ngữ liệu thêm mà các nhóm đã thu thập. Hoạt động này đảm bảo đánh giá chéo giữa các SV (peer assessment). Để giúp cho GV tiện theo dõi hoạt động peer assessment, các e-portfolio đã được thiết kế thêm phần đánh giá và gợi ý chỉnh sửa từ các nhóm do $\mathrm{GV}$ chỉ định. Sau khi nhận được nhận xét, gợi ý chỉnh sửa từ nhóm khác, SV viết lại bản thảo (second draft). Úng dụng Share, Comments, Mode trong Google Docs giúp SV trao đổi e-porfolio và chỉnh sửa các bài viết trong e-porfolio nhanh chóng, dễ dàng. Các bản thảo được viết lại (second draft) được $\mathrm{GV}$ đánh giá, nhận xét. 
- Report (Báo cáo): Sau khi nhận được nhận xét, đánh giá từ GV đối với second draft, SV hoàn chỉnh bản thảo lần cuối (final draft). Sau đó, nhờ ứng dụng Share trong Google Docs, GV sẽ giúp SV công bố e-porfolio cho toàn bộ các nhóm trong lớp, hoặc cho những ai có đường link dẫn tới e-porfolio này.

Giai đoạn củng cố ngôn ngữ (Language Focus) bao gồm hai bước:

- Analysis (phân tích): SV phân tích và thảo luận những đặc trưng ngôn ngữ trong các loại thư tín (cấu trúc thư, cấu trúc ngữ pháp, từ vựng đặc trưng cho mỗi loại thư tín) dưới sự hướng dẫn của GV trên lớp, từ đó rút ra những đặc điểm ngôn ngữ thường gặp trong quá trình hoàn thành từng stage trong simulation task.

- Practice (thực hành): GV cho thêm các bài tập để củng cố từ mới, cấu trúc mới, những kiến thức ngôn ngữ đã được đề cập trong phần Analysis. Hoặc GV có thể đưa ra bài tập viết một dạng thư tín tương tự có trong giáo trình hoặc bên ngoài.

\section{KẾT QUẢ NGHIÊN CÚU}

\subsection{Hiệu quả của việc tích hợp bài tập mô phỏng và đánh giá theo hình thức hồ sơ tài liệu học cá nhân vào việc dạy viết thư tín thương mại}

Sau khi tiến hành thực nghiệm, chúng tôi thu được bảng kết quả điểm của các nhóm. Giá trị thống kê mô tả các kết quả của Pre-test và Post-test được thể hiện trong bảng 1 .

Bảng kết quả trên cho thấy hiệu quả của việc tích hợp bài tập mô phỏng và eporfolio vào viết thư tín thương mại, giúp sinh viên hiểu bài tốt hơn, do đó làm tăng kết quả kiểm tra của sinh viên qua quá trình học. Ban đầu, nhóm đối chứng có kết quả PreTestDC là 5.30 cao hơn kết quả của nhóm thực nghiệm PreTestTN là 4.48, tương ứng là 0.82 điểm. Điều này chứng tỏ nhóm đối chứng học "tốt" hơn nhóm thực nghiệm. Tuy nhiên sau quá trình học mặc dù 2 nhóm đều có sự tiến bộ nhưng kết quả của nhóm đối chứng lại thấp hơn rất nhiều so với nhóm thực nghiệm. Điểm trung bình học tập của hai nhóm đều tăng. Ở nhóm đối chứng, điểm trung bình Pre-test là 5.30, điểm trung 
bình Post-test 1 là 5.35 và Post-test 2 là 5.78 , tương ứng là tăng $1 \%, 9 \%$ (tỉ lệ này xét theo kết quả trung bình của đợt kiểm tra PreTest). Ở nhóm thực nghiệm, điểm trung bình Pre-test là 4.48, điểm trung bình Post-test 1 là 5.99 và điểm trung bình Post-test 2 là 6.91 tương ứng là tăng 33.3\% và $53.3 \%$ (tỉ lệ này xét theo kết quả trung bình của đợt kiểm tra PreTest). Từ kết quả cho thấy: trong đợt kiểm tra PostTest lần 2, điểm của nhóm thực nghiệm tăng tới 53.3\% trong khi nhóm đối chứng chỉ tăng $9 \%$ so với điểm PreTest lần đầu. Điểm tăng trung bình bài kiểm tra PostTest lần 2 của nhóm thực nghiệm là khoảng 2.4 điểm (từ 4.48 lên 6.91), tăng gần 5 lần so với điểm tăng trung bình bài kiểm tra PostTest lần 2 của nhóm đối chứng là 0.48 điểm (từ 5.30 lên 5.78). Do đó, việc áp dụng phương pháp đề xuất giúp cho kết quả học tập của sinh viên tăng nhiều, điều này chứng tỏ hiệu quả của mô hình đề xuất.

Bảng 1. Giá trị thống kê mô tả của các kết quả Pre-test và Post-test

\begin{tabular}{llll}
\hline STT & Giá trị trung bình & Phương sai & Độ lệch chuẩn \\
\hline PreTestDC & 5.30 & 3.63 & 1.90 \\
\hline PostTestDC1 & 5.35 & 3.21 & 1.79 \\
\hline PostTestDC2 & 5.78 & 2.13 & 1.46 \\
\hline PreTestTN & 4.48 & 4.22 & 2.05 \\
\hline PostTestTN1 & 5.99 & 4.97 & 2.23 \\
\hline PostTestTN2 & 6.91 & 1.13 & 1.06 \\
\hline
\end{tabular}

Trong đó: PreTestDC là điểm kiểm tra tiền thực nghiệm của nhóm đối chứng; PostTestDC1 là điểm kiểm tra sau thực nghiệm lần 1 của nhóm đối chứng; PostTestDC2 là điểm kiểm tra sau thực nghiệm lần 2 của nhóm đối chứng; Tương tự PreTestTN, PostTestTN1 và PostTestTN2 cho nhóm thực nghiệm.

Để kiểm tra kết quả thực nghiệm của phương pháp đề xuất có ý nghĩa thống kê, chúng tôi sử dụng phương pháp t-test để kiểm tra 2 giả thuyết sau: 
- Giả thuyết 1: Điểm trung bình kiểm tra PostTest lần 1 và 2 của nhóm thực nghiệm cao hơn điểm trung bình kiểm tra PostTest lần 1 và 2 của nhóm đối chứng.

- Giả thuyết 2: Độ tăng giữa điểm trung bình kiểm tra PostTest lần 1 và 2 với điểm PreTest của nhóm thực nghiệm cao hơn độ tăng giữa điểm trung bình kiểm tra PostTest lần 1 và 2 với điểm PreTest của nhóm đối chứng.

Việc kiểm tra hai giả thuyết 1 và 2 cho giá trị $p$-value tương ứng là 0.01246 và 0.0001759. Cả hai giá trị này đều nhỏ hơn 0.05 . Điều này cho thấy sự khác biệt về kết quả điểm của 2 nhóm có ý nghĩa về mặt thống kê, chứng tỏ sự hiệu quả của phương pháp đề xuất.

\subsection{Kết quả khảo sát thái độ của sinh viên}

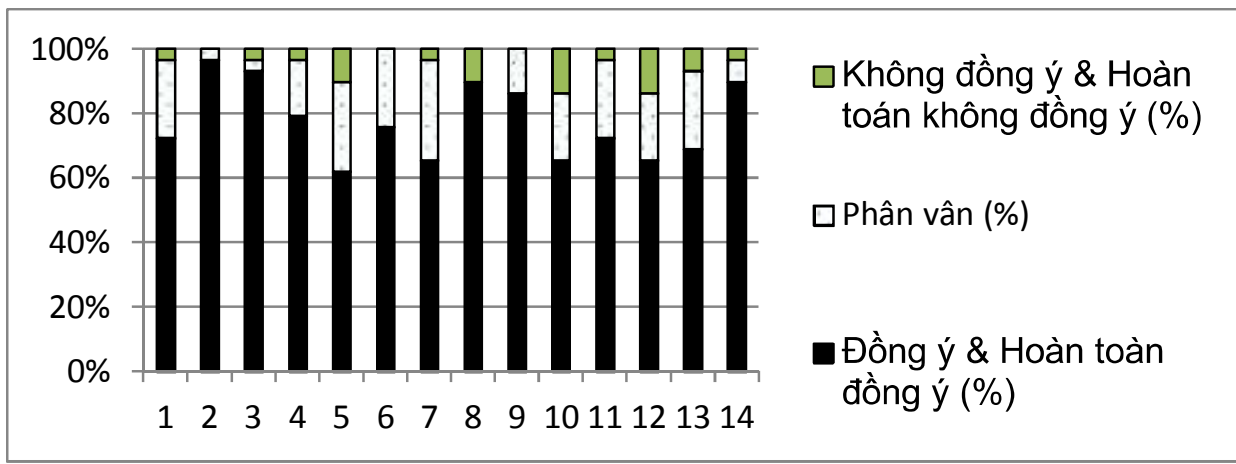

\section{Hình 1. Kết quả khảo sát ý kiến sinh viên}

1. Tôi rất thích các bài tập mô phỏng (simulation task).

2. Giáo viên phổ biến đầy đủ mục đích, yêu cầu các simulation task.

3. Các simulation task có hướng dẫn rõ ràng.

4. Tôi đọc kỹ tài liệu trong giáo trình để hoàn thành các simulation task.

5. Tôi tìm đọc thêm tư liệu bên ngoài giáo trình để hoàn thành các simulation task.

6. Các simulation task giúp tôi hình dung các tình huống giao dịch thương mại rõ ràng, từ đó tôi có thể cân nhắc nội dung $\&$ ngôn ngũ sao cho phù hợp với mỗi loại thư tín trong từng trường hợp cụ thể.

7. Tôi có tham khảo ngữ liệu từ các đường link trong phần Language Focus (nằm cuối eportfolio) để hoàn thành các simulation task. 
8. Tôi luôn thảo luận với bạn cùng cặp, và cùng nhau hoàn thành các simulation task.

9. Hoạt động phân tích ngôn ngữ (Language Focus) giúp tôi mở rộng vốn từ chuyên ngành và ghi nhớ các cấu trúc đặc trưng cho từng thể loại thư.

10. Các e-portfolio tiện lợi cho việc đăng bài, chỉnh sửa bài viết, trao đổi thông tin.

11. Tôi hiểu rõ cách đánh giá một thư tín thương mại theo tiêu chí $5 \mathrm{C}$ 's: Complete, Concise, Clear, Courteous, Correct, và áp dụng phương pháp đánh giá này vào việc đánh giá các bài viết trong e-porfolio một cách hiệu quả.

12. Tôi thấy hoạt động nhận xét, đánh giá e-portfolio giữa các sinh viên giúp tôi cải thiện \& củng cố kiến thức

13. Tôi chỉnh sửa bài viết trong e-porfolio nhiều lần trước khi công bố.

14. Tôi thấy việc tích hợp các simulation task và e-porfolio vào việc học viết thư tín thương mại là có hiệu quả, giúp tôi phát triển kỹ năng viết các loại thư tín thương mại.

Nhằm phân tích thái độ của người học đối với việc sử dụng bài tập mô phỏng và e-portfolio, chúng tôi sử dụng thang độ Likert được đề xuất bởi Likert (1932). Đây là thang đo thái độ của người được nghiên cứu, thông thường được chia làm 5 bậc. Trong bảng câu hỏi khảo sát của nghiên cứu này, chúng tôi đưa ra những câu nhận xét về việc ứng dụng bài tập mô phỏng và e-portfolio và yêu cầu sinh viên thuộc nhóm thực nghiệm cho biết ý kiến của mình về các nhận xét này: hoàn toàn không đồng ý, không đồng ý, phân vân, đồng ý, hay hoàn toàn đồng ý. Số phiếu khảo sát phát ra và thu thập được là 29. Dựa trên kết quả khảo sát thu thập được, kết quả phân tích định lượng cho thấy đa số sinh viên có thái độ tích cực đối với việc tích hợp bài tập mô phỏng và e-porfolio vào chương trình học viết thư tín thương mại. Điều này được thể hiện rõ qua biểu đồ trong hình 1 trình bày kết quả khảo sát ý kiến sinh viên.

Hình 1 thể hiện rõ gần 3/4 số sinh viên được khảo sát cho biết yêu thích các bài tập mô phỏng và gần 9/10 sinh viên đồng ý rằng việc tích hợp các bài tập mô phỏng và e-porfolio vào việc học viết thư tín thương mại là có hiệu quả, giúp phát triển kỹ năng viết các loại thư tín thương mại. Tỉ lệ sinh viên đồng ý các bài tập mô phỏng có hướng dẫn rõ ràng là rất cao $(93.1 \%)$, và giúp sinh viên hình dung các tình huống giao dịch thương mại rõ ràng, từ đó có thể cân nhắc nội dung và ngôn ngữ sao cho phù hợp với mỗi loại thư tín trong từng trường hợp cụ thể (75.9\%). Để hoàn thành bài tập mô phỏng, đa số sinh viên (79.3\%) đọc tài liệu trong giáo trình, và thảo luận với bạn $(89.7 \%)$. Tuy nhiên chỉ gần $2 / 3$ sinh viên cho biết có tìm đọc thêm tư liệu bên ngoài giáo trình, và 
tham khảo tài liệu từ các đường link trong Language Focus. Hơn $86 \%$ sinh viên cho rằng hoạt động phân tích ngôn ngữ trong e-portfolio là hữu ích. Gần 3/4 số sinh viên tự nhận hiểu rõ cách đánh giá, chỉnh sửa một thư tín thương mại theo tiêu chí $5 \mathrm{C}$ 's. Gần $70 \%$ sinh viên thừa nhận có chỉnh sửa bài viết nhiều lần, thể hiện hoạt động tự đánh giá được thực hiện khá thường xuyên. Đáng lưu ý chỉ có gần $2 / 3$ sinh viên đồng ý rằng các e-portfolio tiện lợi cho việc đăng bài, chỉnh sửa bài viết, trao đổi thông tin, và cho rằng hoạt động nhận xét, đánh giá e-portfolio giữa các sinh viên giúp cải thiện, củng cố kiến thức. Khoảng 1/7 sinh viên gặp khó khăn khi thực hiện, chỉnh sửa, trao đổi nhận xét eporfolio trên máy. Điều này có thể liên quan tới việc có khoảng $1 / 3$ sinh viên cảm thấy phân vân hoặc không đồng ý rằng hoạt động nhận xét, đánh giá e-portfolio giữa các sinh viên giúp cải thiện, củng cố kiến thức.

Sau khi phân tích định tính các câu trả lời cho các câu hỏi dạng mở trong bảng câu hỏi khảo sát và phỏng vấn, nguyên nhân vì sao có một số sinh viên không thích việc ứng dụng bài tập mô phỏng và e-porfolio đã được xác định. Có sáu nguyên nhân chính như sau. Thứ nhất, các simulation task khá dài bao gồm nhiều stage. Để hoàn thành các stage này, sinh viên cần tham khảo nhiều chương trong giáo trình, và tư liệu bên ngoài tìm được. Lượng từ vựng lớn, chứa đựng các khái niệm hoàn toàn mới lạ trong chuyên ngành thương mại khiến một số sinh viên vất vả trong việc tìm kiếm và xử lý tư liệu học tập. Thói quen tự học chưa được cao ở một vài sinh viên khiến việc đọc ngữ liệu đầu vào còn nhiều khó khăn. Thứ hai, các e-portfolio trong Google Docs sẽ có dấu hiệu chạy chậm, bị giật khi có dung lượng lớn (từ 70 trang trở lên) nên khiến việc đăng bài, chỉnh sửa, nhận xét của sinh viên đôi lúc không được thuận tiện. Thứ ba, một số sinh viên có ý thức, kỹ năng làm việc theo cặp/nhóm kém làm ảnh hưởng đến tiến độ làm việc của các sinh viên khác. Thứ tư, việc để phần nhận xét, đánh giá chéo giữa các sinh viên, và phần củng cố ngôn ngữ (Language Focus) được thực hiện sau khi các sinh viên đã hoàn thành toàn bộ các stage trong một simulation task khiến một số sinh viên cảm thấy áp lực thời gian, và không thực hiện việc nhận xét, đánh giá chéo, và phân tích ngôn ngữ hiệu quả. Thứ năm, một số sinh viên không có máy tính, hoặc có kỹ năng vi tính kém, làm ảnh hưởng đến việc thực hiện bài trên máy. Thứ sáu, việc sắp xếp thời 
khóa biểu chưa hợp lý nên sinh viên khó tiếp thu hết kiến thức, đôi khi việc xếp lịch học quá dày khiến sinh viên không có thời gian gặp gỡ trao đổi và thực hiện e-portfolio.

\section{MỘT SỐ ĐỀ XUẤT}

Dựa trên kết quả nghiên cứu đã thực hiện, một số đề xuất liên quan tới việc tích hợp bài tập mô phỏng và e-portfolio vào trong quá trình dạy viết thư tín thương mại được đưa ra dưới đây. Những đề xuất này được trình bày tương ứng với kết quả khảo sát ý kiến sinh viên thu thập được đề cập trong phần 4.2 bên trên.

Kết quả khảo sát ý Đề xuất kiến sinh viên

Các simulation task Không nên để sinh viên làm xong toàn bộ các stage trong một khá dài bao gồm bài tập mô phỏng, rồi mới thực hiện việc trao đổi e-porfolio nhiều stage. để đánh giá chéo giữa các sinh viên. Việc đánh giá chéo giữa các sinh viên nên được thực hiện ngay sau khi hoàn thành một stage, sau đó sinh viên phân tích ngôn ngữ và làm thêm các bài tập để củng cố từ vựng và cấu trúc (Language focus), trước khi chuyển qua stage tiếp theo, và tiếp tục như vậy cho đến hết bài tập mô phỏng.

Các e-portfolio trong Google Docs sẽ có dấu hiệu chạy chậm, bị giật khi có dung lượng lớn.

Một số sinh viên có ý thức, kỹ năng làm việc theo cặp/nhóm kém.

Phần nhận xét, đánh giá chéo giữa các sinh viên, và phần củng cố ngôn ngữ được thực hiện sau khi hoàn thành toàn bộ các stage trong một simulation task khiến một số sinh viên cảm thấy áp lực thời gian.
Nên thiết kế các e-porfolio của các nhóm sinh viên thành các file Google Docs riêng lẽ, không nên để tất cả các e-porfolio của toàn bộ các nhóm trong một file Google Docs. Như vậy sẽ tránh được tình trạng file quá nặng, chạy chậm, gây khó khăn cho sinh viên làm bài, chỉnh sửa, đưa nhận xét.

Cần giám sát mức độ tham gia của sinh viên trong quá trình mô phỏng. Nâng cao ý thức làm việc nhóm trong sinh viên. Nên có các buổi nghe ý kiến của sinh viên định kỳ sau giờ học để nắm được các khó khăn sinh viên gặp phải, từ đó có những điều chỉnh kịp thời, hợp lý.

Đối với một số chương chứa đựng nhiều khái niệm mới và có thể gây khó khăn cho sinh viên trong quá trình xử lý ngữ liệu đầu vào, giáo viên có thể đưa phần Language Focus lên trên, kết hợp vào giai đoạn Pre-task. Sinh viên dưới sự hướng dẫn của giáo viên thực hiện phân tích ngôn ngữ trong các bức thư mẫu, nhờ đó nắm được từ vựng, các cấu trúc cần thiết để hoàn thành bài tập mô phỏng.

Một số sinh viên


không có máy tính, ảnh hưởng đến kết quả học tập. Do đó giáo viên và khoa nên hoặc có kỹ năng vi khuyến khích, tạo điều kiện cho các sinh viên này đạt được kỹ tính kém. năng vi tính cần thiết.

Việc sắp xếp thời Thời khóa biểu dành cho môn Tiếng Anh thương mại 4: khóa biểu chưa hợp lý Thương mại quốc tế nên được kéo dãn ra, mỗi tuần một buổi. nên sinh viên khó tiếp Muốn như vậy thì thời gian bắt đầu học nên từ cuối tháng 8 , thu hết kiến thức. khi bắt đầu học kỳ mới. Với lịch học dàn trãi như vậy, sinh viên mới có đủ thời gian đọc và phân tích tài liệu trong và ngoài giáo trình, và làm thêm được nhiều bài tập mô phỏng hơn. Nhờ vậy, hoạt động trao đổi e-portfolio và đánh giá chéo giữa các sinh viên có thể được thực hiện tốt hơn và có hiệu quả hơn, đồng thời giảm tải áp lực thời gian hoàn thành bài cho sinh viên.

\section{KẾT LUẬN}

Kết quả nghiên cứu cho thấy nên tích hợp các bài tập mô phỏng và e-portfolio cùng một lúc trong việc dạy viết thư tín thương mại vì giúp sinh viên tái tạo ngôn ngữ trong ngữ cảnh gần với thực tế. Sinh viên có thể liên tục kiểm chứng sự phát triển ngôn ngữ bản thân thông qua toàn bộ quá trình học, từ đó tích cực chủ động học tập, nghiên cứu, và phát triển kỹ năng viết thư tín thương mại. Phải đảm bảo các bài tập mô phỏng là phù hợp với yêu cầu phát triển ngôn ngữ trong từng giai đoạn, đồng thời mô phỏng gần với thực tế, để tạo hứng thú nơi người học.

Phương pháp đề xuất có khả năng ứng dụng cao trong các lớp dạy viết thư tín thương mại vì cung cấp cho người dạy qui trình thực hiện e-portfolio đã được mô tả chi tiết trong mục 3.7 trong bài viết này và mẫu e-portfolio ứng dụng trên tệp tin Google.docs được thiết kế sẵ.

Mặc dù nghiên cứu này đã được tiến hành trên nền tảng cơ sở lý thuyết vững chắc, có kết quả thực nghiệm và kết quả khảo sát ý kiến sinh viên khả quan, nhưng vẫn còn một số nhược điểm trong nghiên cứu. Cụ thể, các đề kiểm tra Pre-test và Post-test có thể chưa phản ánh hết quá trình học tập của SV. Thứ hai, kết quả kiểm tra trước và sau thực nghiệm có thể chưa thật chính xác, vì các kết quả này có thể bị ảnh hưởng bởi yếu tố chủ quan của giáo viên tham gia kiểm tra đánh giá. 
Tuy vậy, kết quả t-test trong nghiên cứu này đã cho thấy sự khác biệt về kết quả điểm của 2 nhóm có ý nghĩa về mặt thống kê. Điều này chứng tỏ việc áp dụng phương pháp đề xuất và không áp dụng phương pháp đề xuất cho ra kết quả khác nhau, cụ thể là phương pháp thực nghiệm cho ra kết quả cao hơn và giúp sinh viên học có hiệu quả hơn so với phương pháp kiểm chứng. Do đó, có thể áp dụng phương pháp đề xuất cho các lớp tiếng Anh thương mại 4 trong tương lai, hoặc những lớp tiếng Anh chuyên về viết thư tín thương mại.

\section{TÀI LIỆU THAM KHẢO}

[1] Archibald, D.A. \& Newmann, F.M. (1992). Approaches to Assessing Academic Achievement. In H. Berlak et al. (Eds.), Toward a New Science of Educational Testing and Assessment. Albany. NY: State University of New York Press.

[2] Cambridge, B. L. (2001). Electronic Portfolios as Knowledge Builders. In B.L. Cambridge et al. (Eds.), Electronic Portfolios: Emerging Practices in Student, Faculty, and Institutional Learning. Washington, DC: American Association for Higher Education.

[3] Đào, T.T.P. (2014). Vận Dụng Phương Pháp Dạy Học Theo Nhiệm Vụ Trong Tiến Trình Giảng Dạy Ngoại Ngữ - Công Đoạn Xây Dựng Hoạt Động Dạy Học. Ngôn Ngũ \& Đời Sống, 12 (230), 77-81.

[4] Ellis, R. (2003). Task-based language learning and teaching. Oxford: Oxford University Press.

[5] Ferrari, L. \& Zhurauskaya, D. (2012). E-Portfolios for Language Learning and Assessment. 5 th International conference "ICT for Language learning and Assessment".

[6] Hamp-Lyons, L. \& Condon, W. (1998). Assessing the Portfolio: Principles for Practice, Theory, and Research. Cresskill, NJ. Hampton Press.

[7] Hu, R. (2013). Task-Based Language Teaching: Responses from Chinese Teachers of English. The Electronic Journal for English as a Second Language, 16(4).

[8] Jeevaratnam, G. (2013). Portfolio as an Assessment Tool in French Foreign Language Classroom. International Journal of Scientific Research, 2 (11), 155-156.

[9] Jones, L. \& Alexander, R. (2000). New International Business English. Cambridge: Cambridge University Press.

[10] Lee, J. (2000). Tasks and Communicating in Language Classrooms. Boston: McGraw-Hill. 
[11] Likert, R. (1932). A Technique for the Measurement of Attitudes. Archives of Psychology, 140, 1-55.

[12] Littlejohn, A. (2005). Company to Company: A task-based approach to business emails, letters and faxes. Cambridge: Cambridge University Press.

[13] Milanovic, M. \& Saville, N. (1996). Introduction. In M. Milanovic and N. Saville (Eds.), Performance Testing, Cognition, and Assessment. Cambridge: Cambridge University Press.

[14] Nguyen, V. H. (2014). Review of Notion and Framework of Task-Based Language Teaching. International Journal of English Language and Linguistics Research, 2 (1), 39-48.

[15] Sampath, D. \& Zalipour, A. (2009). Practical Approaches to the teaching of Business English. Proceedings of the $2^{\text {nd }}$ International Conference on Teaching and Learning (ICTL 2009). INTI University College, Malaysia.

[16] Wang, M. (2003). Business Communication Teaching Reform: A Tentative Study of Sino-Finnish International E-mail Project.

[17] Willis, J. (1996). A framework for task-based learning. Harlow, U.K.: Longman Addison- Wesley. 


\title{
INTEGRATING SIMULATION TASKS AND PORTFOLIO ASSESSMENT INTO WRITING BUSINESS CORRESPONDENCE
}

\author{
Hua Thi Tin ${ }^{\text {a* }}$ \\ ${ }^{a}$ The Faculty of Foreign Languages, Dalat University, Lamdong, Vietnam \\ "Corresponding author: tinht@dlu.edu.vn \\ Article history \\ Received: December $03^{\text {rd }}, 2015$ \\ Received in revised form $\left(1^{\text {st }}\right)$ : March $15^{\text {th }}, 2016 \mid$ Received in revised form $\left(2^{\text {nd }}\right)$ : March $25^{\text {th }}, 2016$ \\ Accepted: March $29^{\text {th }}, 2016$
}

\begin{abstract}
This paper presents the results of integrating simulation tasks and portfolio assessment into writing business correspondence in Business English classes at the Faculty of Foreign Languages, Dalat University, and investigates students' attitude toward this proposed method in the practice of writing business correspondence. This study was carried out based on the theory of task-based language teaching with the use of simulation tasks, and on the theory of portfolio assessment. The results reveal that this proposed method using eportfolios (electronic portfolios) benefited the participants although some shortcomings arising from the experimental process should be adjusted and carefully considered in order to achieve better results.
\end{abstract}

Keywords: Business letters; Portfolio assessment; Task-based language teaching. 